\title{
Bayesian probabilistic models for corporate context, with an application to internal audit activities
}

\author{
Francesco Toraldo $^{*} \quad$ Fabio S. Priuli ${ }^{\dagger}$
}

October 5, 2021

\begin{abstract}
In this paper we present a business case carried out in Poste Italiane, in the context of fair performance evaluations of human resources engaged in internal audit activities. In addition to the development of a Bayesian network supporting the goal of the Internal Audit unit of Poste Italiane, the work has led to the development of a methodological approach to advanced analytics in corporate context, whose usefulness goes well beyond the specific use case described here. We thus present the different stages of such analytical strategy, from feature selection, to model structure inference and model selection, as a general toolbox that allows a completely transparent and explainable process to support data-driven decisions in business environments.
\end{abstract}

\section{Introduction}

Data analytics and machine learning represent a resource with ever growing presence in corporate context. Mainstream media interest seems to be captured only when a new advancement is made in the field of computer vision and image recognition, or when an intelligent algorithm defeats humans in some board games, or when a scandal uncovers misuses of private data collected by big IT companies. In fact, the potential contribution of data science in moving corporate decisional processes towards a data-driven path has made them an invaluable tool in a much wider variety of contexts: from capacity planning to fraud management, from real time production monitoring to autonomous driving and controlling systems.

In this article, we propose an (almost) end-to-end chain of data-driven solutions, designed to support the various phases of typical corporate decision making processes, through an extensive usage of Bayesian probabilistic tools. Such methodological framework allows to treat a wide range of business processes with almost no need of ad hoc adjustment, while ensuring coherent evaluations of uncertainty and enabling several kinds of what if analysis. Our proposed framework is the byproduct of a series of projects developed by the Internal Audit department of Poste Italiane in 2015-2019, in strict collaboration with data scientists from an external advisory company Pangea Formazione, now part of Bain \& Company. Each project was built on top of the earlier ones, expanding the scope of the probabilistic forecast to cover further analysis, which allowed, in turn, more informed decisions. In particular, the activities developed in the last year led to the completion of the framework we present here: a few software solutions which could support effectively the whole decision making process, from the selection of relevant variables, to the formulation of a suitable

*Poste Italiane S.p.A., Rome, Italy

${ }^{\dagger}$ Bain \& Company Inc., Rome, Italy 
statistical model of the process of interest, to the actual inference of parameters through some of the most advanced statistical techniques available, and to the prediction of variables of interest.

In Section 2 we will give a detailed outline of a motivating application, taken from the business case that involved experts from the Internal Audit unit at Poste Italiane: a tool to monitor performance evaluations of auditors based on past evaluations of "similar" auditors, so to highlight possible deviations and personal biases in such evaluations and, in case, to ensure more fair judgements. However, it shall be clear that the same basic idea could be applied well beyond this specific example: Poste Italiane itself has applied this approach in multiple internal audit activities and to monitor risk impacts of changes in procedures and controls; and Pangea Formazione has implemented analogous tools in a variety of corporate contexts, from capacity planning to marketing strategies, from predictive maintenance to retention and anti-churn activities.

In general, in presence of complex corporate procedures and activities, Bayesian tools allow to synthetize all available information and to exploit them in a consistent framework, while retaining the capability to inspect in depth the reasons behind each evaluation (through the inspection of specific marginal distributions, given some evidences and observations). This offers a huge advantage, in large business activities, compared to other machine learning solutions like boosting trees or deep neural network which act more like "black boxes", because it gives decision makers a mean to explain the rationale behind their decisions. At the same time, the procedure does not completely automatize the chain of operations: the quantitative results provided at each step shall be (and typically are) reviewed by human experts, to monitor the possible presence of biases in the collected samples of data, or to integrate effects that experts know are present but are for some reason hard to observe in the data available. This integration of human and algorithmic capabilities tries to take the best of both worlds (AI as augmented intelligence rather than artificial intelligence) and it allows to validate the proposals of each algorithm through the experience of people who work daily on the processes of interest.

In the following sections, we will give more details about the four different phases that constitute our approach and that provide support to different stages of corporate decision making processes:

- Phase 1 - variable selection based on the concepts of mutual information and conditional mutual information;

- Phase 2 - structure inference of Bayesian networks for the process of interest, combinining the variables selected in the earlier phase with experts' proposals;

- Phase 3 - model comparison of the more promising models through Bayes-Turing factor, and comparison with a baseline benchmark (typically a naïve Bayes classifier, if we are dealing with discrete classification problems);

- Phase 4 - parameter inference and predictions through Markov chain Monte Carlo simulations ( $M C M C$ in the following).

While each step is not original, the actual application of the whole procedure in corporate contexts is far from common, to our knowledge, and no commercial data analysis or statistical platform seems to implement it organically. Thus, in the projects at Poste Italiane, we developed our own tools in the form of scripts written in the statistical programming language $\mathrm{R}$ [1], by incorporating several of its statistical libraries [2, 3, 4, and by exploiting for the MCMC simulations the JAGS library, through its R interface [5]. Such a solution allows a flawless integration in every business workflow where it is allowed to call external command line scripts as an intermediate stage, with resulting structures and data saved to temporary files. 


\section{An example of corporate application}

The Internal Audit group in Poste Italiane has been using Bayesian modelling techniques to perform quantitative evaluation and scenario analysis since a few years. They applied such techniques in very different contexts, both as a tool to turn different sources of evaluations into more coherent indicators and as a tool to forecast multiple KPIs for audit activities, based on the characteristics of each post office and of each team of auditors.

Bayesian techniques are invaluable in corporate processes, because they allow to exploit all available information, usually quite sparse, and to synthetize it consistently with experts' opinions that can be modelled, not without considerable efforts, in forms of prior distribution of probability to be inserted into the model.

In this section, we present a concrete example in which our proposed end-to-end procedure was applied at Poste Italiane. Namely, we built a Bayesian model which aimed to support performance evaluations of human resources engaged in internal audit activities, after having learnt from historical data what kind of evaluation 'similar' auditors had received. The construction was performed through the steps outlined in Section 1; by selecting relevant variables through their mutual information, by inferring different Bayesian networks structures and comparing them through subject matter's opinions and the Bayes-Turing factor $B F$ (whose logarithm is also known as the "weight of evidence"), by performing predictions of the quantity of interest through MCMC simulations.

The plan of this section is, therefore, to start describing the context and the relevant features that determine the quantities at play, then to move to describe each step of the construction in more details. Since we are not adding new statistical developments, we defer to the Appendix 4 the description of some of the statistical tools and techniques used throughout the process.

A few key aspects of the construction are the following:

- for all network structures, we make the same assumptions on the data-generating process by assigning a Categorical distribution to each feature, and assuming their parameters are $a$ priori uniformly distributed;

- we compare structure learning algorithms which are built by optimizing different scores (BIC, likelihood or BDeu), by using both $B F(6)$ and the predictions on a separate test set (to measure latter predictive performances, we use root of mean square error because we primarily aim to small errors);

- in the application at hand, the model selection phase through $B F$ does not lead directly to the selection of a single candidate model: since we have only a few hundreds of data records available, in order to mitigate the risk of overfitting, we keep all models that perform better than a naïve Bayes classifier, and then we perform a 10-fold cross validation procedure, along the lines of [18], to make our final choice.

In view of the focus on categorical features, we make large use of information measures (like Shannon entropy and mutual information) both to select variables to be included in the model, and to review different network structures, in search for links that could be safely removed from the model. In presence of continuous features, or mixtures of both continuous and discrete variables, such quantities require to assume a suitable model of the joint distribution of pairs of variables. When subject matter experts want to avoid such a modelling step, it becomes important to exploit other available "correlation" measures or selection approaches: e.g. by using nonparametric sure independence screening [19, 20] to decide which features can be discarded; by choosing suitable prior distributions to continuous parameters, so to penalize models with large parameters or too many variables, like with ridge or LASSO regularization [21]; by measuring the impact of each 
independent variable on the target through partial dependency plots, or ICE plots, similarly to what could be done with black box models [22]. We do not account for these alternative approaches in this paper.

\subsection{Corporate context: internal audit activities}

The motivation behind the construction of a model for performance evaluations of human resources is to ensure more homogeneous judgements, through identification of possible biases in the auditors' evaluations. Namely, a predictive model that suggests an appropriate score to different auditor profiles is expected to provide a solid anchor to compare further evaluations with, so to avoid the risk of making them too subjective. Evaluations given by the model would not be prescriptive, but they would help to highlight deviations from the past judgements, making possible to analyse whether such deviations are really motivated by changes in the priorities of the corporation, that might e.g. decide at some point to give priority to reward more innovation skills, or if it is affected by external factors that should not have influence. Most often the reason for non-conforming evaluations is a combination of different factors, and the model can be used to raise an alarm whenever there is a difformity that shall be better understood.

In order to decide what kind of profile each auditor would belong to, we use a set of nine predictors, extracted from the Leadership model used in Poste Italiane and linked to the conceptual category of "abilities". Such predictors represent measures for the evaluation of all workers in Poste Italiane, not only auditors. They are in detail:

- Value for the customer: a measure of the centrality of the customers in the activities, based on the rapidity of the services offered and the attention to customer's needs;

- Innovation and simplification: a measure of the capacity to exploit innovative approaches and to simplify procedures, while keeping a high level of quality;

- Vision: a measure of the capacity to read the whole context in which daily activities are embedded, acknowledging interdependencies among processes and their complexity, while suggesting solutions that could help operativity;

- Propensity to change: a measure of the capacity to adopt new operative models that are being progressively introduced in the company, and to support transformations through direct involvement and collaboration;

- Decision-making ability: a measure of the skills related to properly assessing risks and future scenarios and to effectively managing uncertainties in a consistent and appropriate way;

- Inclusive leadership: a measure of the team leading ability and the level of involvement established with colleagues, collaborators and customers;

- Integrity: a measure of the respect of ethical principles with coherent behaviors both in daily operativity and in the interactions with customers and colleagues;

- Collaboration and ability to influence: a measure of the capacity to cooperate with other business units in order to improve and speed up decision making processes;

- Resource Engagement: a measure of the level of engagement and proactivity of the resource in her daily job.

In the leadership model, these quantities are evaluated as an integer number in the range 1 to 10. However, when used in the evaluation of auditors, the features are discretized into quintiles in order to facilitate their handling. Based on historical data, Internal Audit experts have chosen 5 thresholds for each features, so that data records are split into 5 equally numerous buckets. Then, 
present and future auditors are assigned an integer score from 1 to 5 depending on the interval their numerical score belongs to: 1 if they are in the top 20\% of the evaluations, and then decreasing towards 5 if they are in the bottom $20 \%$.

The target variable for the model is then the "global evaluation" for a new auditor (named Final evaluation in our dataset), based on its characteristics and attitudes, as an integer in a scale from 1 to 10, with 1 being the best value and 10 being the worst one.

For the analysis, we had historical data related to 234 auditors: the small number is due to the need for only using fresh data in the model, because of the moving objectives of the criteria used, which tend to be refined as time goes. As a result, evaluations of skills from a few years ago are not really comparable with newer ones. This restriction has an unfortunate side effect, in this specific application, because the target variable, even though it is supposed to span from 1 to 10 , is only present with values in 1 to 8 because no records with the worst scores are included. This means that the model is not expected to have high precision for very negative cases, and that MCMC simulations will often require more iterations to reach convergence for the target Dirichlet distributions.

\subsection{Variable selection}

The first step consists of selecting the most promising predictors, based on mutual information scores (both unconditional and conditional) as defined in (5). A series of $\mathrm{R}$ scripts, applied to our original dataset, allows to create different scoring tables like in Table 1 ;

- a table with the values of $\mathrm{MI}^{\prime}(X, Y)$, for each pair of predictors $(X, Y)$, sorted by decreasing scores (see Table 1-top);

- a table with the values of $\mathrm{CMI}^{\prime}(X, Y \mid Z)$, for each triple of predictors $(X, Y, Z)$, sorted by decreasing scores (see Table 1 -middle);

- a table with the comparison among the scores $\operatorname{MI}^{\prime}(X, Y)$ and $\mathrm{CMI}^{\prime}(X, Y \mid Z)$, so to highlight situations where pairs of variables which would usually be dropped, could instead be highly informative, when their distribution is considered as conditional on a third variable (see Table 1-bottom).

They also provide histograms of the distributions of $\mathrm{MI}^{\prime}$ and $\mathrm{CMI}^{\prime}$ (cfr. Figure 1), so to effectively support the understanding of how each score is positioned in the overall distribution and the possible choice of an acceptance threshold to drive feature elimination.

Recalling that at this stage we are mostly interested into information scores from (5), so to select which features to include in our predictive model, we focus our attention to the bottom of the $\mathrm{MI}^{\prime}$ table, where less informative variables are located, and we analyse their contribution. In this specific case, the variable which have smallest mutual information with the others is Resource

Engagement, which is responsible for the tail of the histogram of $\mathrm{MI}^{\prime}$ in Figure 1-left. Before deciding whether to drop it, though, we want to check also the possible increase observed when the distribution of Resource Engagement is conditional on other variables, i.e. its CMI' score. This is indeed the case, as one can see in Table 1 -bottom: $\mathrm{CMI}^{\prime}(X$, Resource Engagement $\mid Z)$ do increase when compared to $\mathrm{MI}^{\prime}(X$, Resource Engagement), for many choices of variables $X$ and $Z$. However, the values of $\mathrm{CMI}^{\prime}$ remain too small compared to the ones of the other variables, available on Table 1-middle, and thus do not seem to justify the inclusion of the variable Resource Engagement in the model. Similarly, focusing the $\mathrm{MI}^{\prime}-\mathrm{CMI}^{\prime}$ comparison to the case when Resource Engagement is the conditioning variable, as shown in Table 2, we get an increase in $\mathrm{CMI}^{\prime}$, but the variation compared to the corresponding $\mathrm{MI}^{\prime}$ seems too small to justify the inclusion of the variable. 
Table 1: Sample of rows from the tables containing mutual information scores (5) which are relevant for our feature selection process: the first table contains the $\mathrm{MI}^{\prime}$ score for each pair of features, in decreasing order; the second table lists the $\mathrm{CMI}^{\prime}$ score for each triple of features, in decreasing order; finally, the third table presents the variation (delta and perc) between $\mathrm{MI}^{\prime}$ and $\mathrm{CMI}^{\prime}$ when one assumes conditioning on a third variable.

\begin{tabular}{ll}
\hline pair & $\mathrm{MI}^{\prime}$ \\
\hline $\mathrm{MI}^{\prime}($ PROP_TO_CHANGE, COLLAB_INFL) & 0.281 \\
MI'(LEAD_INCL, COLLAB_INFL) & 0.271 \\
MI'(VISION, PROP_TO_CHANGE) & 0.27 \\
MI'(PROP_TO_CHANGE, LEAD_INCL) & 0.25 \\
MI'(VISION, COLLAB_INFL) & 0.249 \\
MI'$^{\prime}($ PROP_TO_CHANGE, DEC_MAKING) & 0.247 \\
$\ldots$ & $\ldots$ \\
\hline
\end{tabular}

\begin{tabular}{|c|c|}
\hline triplet & $\mathrm{CMI}^{\prime}$ \\
\hline $\mathrm{CMI}^{\prime}\left(\mathrm{PROP}+\mathrm{TO} \_\mathrm{CHANGE}, \mathrm{COLLAB} I N F L-\right.$ RES_ENG $)$ & 0.3 \\
\hline CMI'(VISION, PROP_TO_CHANGE — RES_ENG) & 0.288 \\
\hline $\mathrm{CMI}^{\prime}(\mathrm{LEAD} I \mathrm{INCL}, \mathrm{COLLAB} I \mathrm{INFL}-\mathrm{RES} E \mathrm{ENG})$ & 0.274 \\
\hline CMI'(PROP_TO_CHANGE, DEC_MAKING - RES_ENG) & 0.271 \\
\hline CMI'(PROP_TO_CHANGE, LEAD_INCL - RES_ENG) & 0.266 \\
\hline CMI'(INNOV_SIMPL, LEAD_INCL - RES_ENG) & 0.261 \\
\hline ... & $\cdots$ \\
\hline
\end{tabular}

\begin{tabular}{lllllll}
\hline $\mathrm{x}$ & $\mathrm{y}$ & $\mathrm{z}$ & $\mathrm{CMI}^{\prime}$ & $\mathrm{MI}^{\prime}$ & delta & perc \\
\hline INTEGRITY & RES_ENG & VAL_FOR_CLI & 0.048 & 0.026 & 0.021 & $80.3 \%$ \\
VAL_FOR_CLI & RES_ENG & INTEGRITY & 0.044 & 0.024 & 0.019 & $79.1 \%$ \\
INNOV_SIMPL & RES_ENG & INTEGRITY & 0.039 & 0.023 & 0.016 & $68.3 \%$ \\
INTEGRITY & RES_ENG & DEC_MAKING & 0.044 & 0.026 & 0.018 & $66.4 \%$ \\
INTEGRITY & RES_ENG & INNOV_SIMPL & 0.044 & 0.026 & 0.017 & $65.1 \%$ \\
DEC_MAKING & RES_ENG & INTEGRITY & 0.043 & 0.027 & 0.016 & $59.4 \%$ \\
$\ldots$ & $\ldots$ & $\ldots$ & $\ldots$ & $\ldots$ & $\ldots$ & $\ldots$ \\
\hline
\end{tabular}



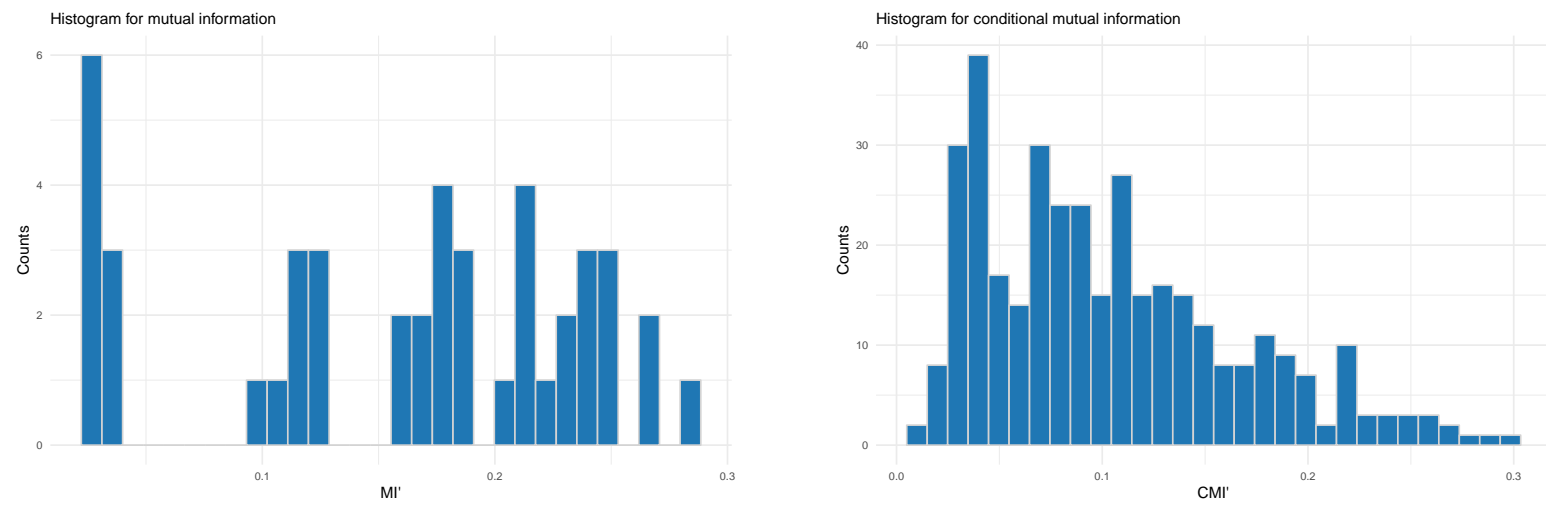

Figure 1: Distributions of normalized mutual information scores (5), unconditional (left) and conditional (right), can help the choice of an acceptance threshold in the feature selection process.

Table 2: Before discarding a feature (e.g. Resource Engagement), one shall check the conditional mutual information scores (5), to be sure that such variable does not play a relevant role as parent node of other features: this can be evaluated by examining the variations between $\mathrm{MI}^{\prime}$ and $\mathrm{CMI}^{\prime}$.

\begin{tabular}{lllllll}
\hline $\mathrm{x}$ & $\mathrm{y}$ & $\mathrm{z}$ & $\mathrm{CMI}^{\prime}$ & $\mathrm{MI}^{\prime}$ & delta & perc \\
\hline VAL_FOR_CLI & INTEGRITY & RES_ENG & 0.126 & 0.097 & 0.029 & $29.8 \%$ \\
INNOV_SIMPL & INTEGRITY & RES_ENG & 0.129 & 0.104 & 0.025 & $23.8 \%$ \\
DEC_MAKING & INTEGRITY & RES_ENG & 0.152 & 0.127 & 0.025 & $19.9 \%$ \\
VAL_FOR_CLI & PROP_TO_CHANGE & RES_ENG & 0.176 & 0.156 & 0.02 & $12.8 \%$ \\
INTEGRITY & FINAL_EVAL & RES_ENG & 0.129 & 0.115 & 0.014 & $12.4 \%$ \\
PROP_TO_CHANGE & INTEGRITY & RES_ENG & 0.142 & 0.127 & 0.015 & $11.8 \%$ \\
$\ldots$ & $\ldots$ & $\ldots$ & $\ldots$ & $\ldots$ & $\ldots$ & $\ldots$ \\
\hline
\end{tabular}


Therefore, domain experts concluded against the inclusion of Resource Engagement in view of model parsimony.

\subsection{Structure learning and model comparison}

Once the feature selection phase is completed, we move to the second stage of our analysis, consisting of training several structure algorithms over the available dataset. The $\mathrm{R}$ scripts we use can automatically produce a series of reports for the expert to analyse. Such reports typically include, for each available algorithm, the following components:

- proposal of network structure, as those in Figure 2;

- a sensitivity analysis of the target variable w.r.t. changes in every other predictors in the proposed model, as those in Table 5 .

Here, by "sensitivity analysis" we mean a quantitative score given to the amount of information that each predictor $V$ carries about the target variable $T$. An appropriate measure of such an impact is given by the variation in entropy (2) due to the collected evidences, which we call sensitivity $\mathcal{S}(T, V)$ and define as:

$$
\mathcal{S}(T, V)=H(T)-H(T \mid V) .
$$

By measuring this quantity, experts can decide which connections to remove if the resulting network structure is still too rich of connections and thus too computationally expensive: the links between $T$ and $V$ with very small $\mathcal{S}(T, V)$ can be removed. Notice that in general the value $\mathcal{S}$ is conditional on the graph structure $\mathcal{G}$ of the Bayesian network as well, even if we do not make this dependence explicit through a notation like $\mathcal{S}_{\mathcal{G}}$.

In this application, we chose to focus our attention on a restricted selection of the available structure learning algorithms in Scutari's bnlearn package [2, Mihaljevic's bnclassify [3] and Suzuki's BNSL [4]. Namely, we will compare the structures proposed from:

- "Hill climbing" greedy algorithm from bnlearn, denoted with hc in the following, which searches for a structure maximizing the Bayesian Information Criterion or BIC (see [2] for details);

- "Tree augmented network" algorithm from bnclassify, denoted with tan in the following, which maximizes likelihood as described in the paper by Friedman et al. (see [23] for details);

- "BNSL" algorithm from the package with the same name, denoted with bnsl in the following, which contains Suzuki's implementation of Chow-Liu's algorithm and searches for structures which maximize a Bayesian Dirichlet (BD) score (see [4] for details).

Most of these algorithms allow to enforce the presence of specific connections among variables or to ensure that some link should not be included. Some alternative tools also allow for the inclusion of logical constraints among variables or penalizations of larger cliques (see e.g. HuginLite in [24]), if such input from expert is deemed worth the inclusion. In the present case, we did not use any of these, and we preferred instead to include a couple of additional network structures which experts wanted to put under test:

- a modified version of the hc structure, where we invert the direction of the arrow connecting the variables Final evaluation and Inclusive leadership, denoted with hc mod in the following; 
Table 3: Sample of rows from the table containing the (logarithm of) BF scores (6), for each pair of models (left) or for each model against naïve structure (right). The larger is $\mathrm{BF}(\mathrm{A}, \mathrm{B})$, the most probable is model A compared to model B, given the available dataset.

\begin{tabular}{lll}
\hline model 1 & model 2 & $\log (\mathrm{BF})$ \\
\hline hc & hc_mod & 105.977 \\
bnsl & hc_mod & 100.54 \\
hc & tan & 97.428 \\
$\cdots$ & $\cdots$ & $\cdots$ \\
\hline
\end{tabular}

\begin{tabular}{lll}
\hline model 1 & model 2 & $\log (\mathrm{BF})$ \\
\hline hc & naive & 96.529 \\
bnsl & naive & 91.092 \\
naive & hc_mod & 9.448 \\
$\ldots$ & $\ldots$ & $\ldots$ \\
\hline
\end{tabular}

- the undirected network structure proposed by "Chow-Liu algorithm" in bnlearn, with directions for the links added by the experts of the process, based on their experience and on some hints from the $\mathrm{MI}^{\prime}$ and $\mathrm{CMI}^{\prime}$ scores previously computed, and denoted with chowliu in the following.

Notice that, by changing some of the configuration options (= hyperparameters) in the algorithms above, e.g. the network score in hc or tan, we might obtain slightly different network structures, that shall be validated by domain experts, and possibly added to the list of models to be evaluated in the following. In the specific application considered here, though, no appreciable differences resulted by different choices of the hyperparameters.

In Figure 2 the five resulting network structures are shown side-by-side for comparison, together with the structure of the naïve Bayes classifier. In both such additional structures the target variable, Final evaluation, appears only as parent node for some of the remaining variables. This is in line with the idea of using measurable characteristics to infer the features that cannot be directly measured, like the target one here. Such a choice also helps to keep under control the size of the conditional probability tables $(\mathrm{CPT})$ of the target variable, which is important in this specific example because of the small size of the available dataset: as the number of parent nodes of a variable increases, indeed, so does the number of parameter in its CPT, and therefore we risk to have not enough data to learn their correct distributions.

Once we have a series of DAG proposals, our scripts compute Bayes-Turing factors (6) for each pair of models, and between the learned structures and the naïve one, as in Table 3. BFs can then be turned into an ordered hierarchy, as shown in Table 4 , so to conclude that hc and bnsl are definitely more capable to describe the dataset, compared to the other algorithms. However, in this specific example, we had a very small dataset and we were worried that the observed BFs might be affected by some overfitting phenomena: recall that, due to its definition, the Bayes-Turing factor selects the best DAG structure by itself, only when we can assume a uniform probability distribution among DAG structures. When domain experts find difficult to discriminate a priori among different structures, we shall be careful in relying on the dataset alone for selection. Here, we decided to exploit BF only to drop the model hc_mod from the analysis, since it scored definitely less than a naïve structure, and to postpone the final selection after a comparison of the predictive performances of the remaining four models through a cross-validation procedure, which will be the focus of next section.

Before moving to the next phase, however, we want to stress that even at this stage it is possible to perform an analysis of interest. Indeed, we can help experts in verifying their beliefs on the importance of different features for the task at hand, against the actual sensitivity analysis offered by each network structure. According to experts, the Final evaluation of auditors' job 

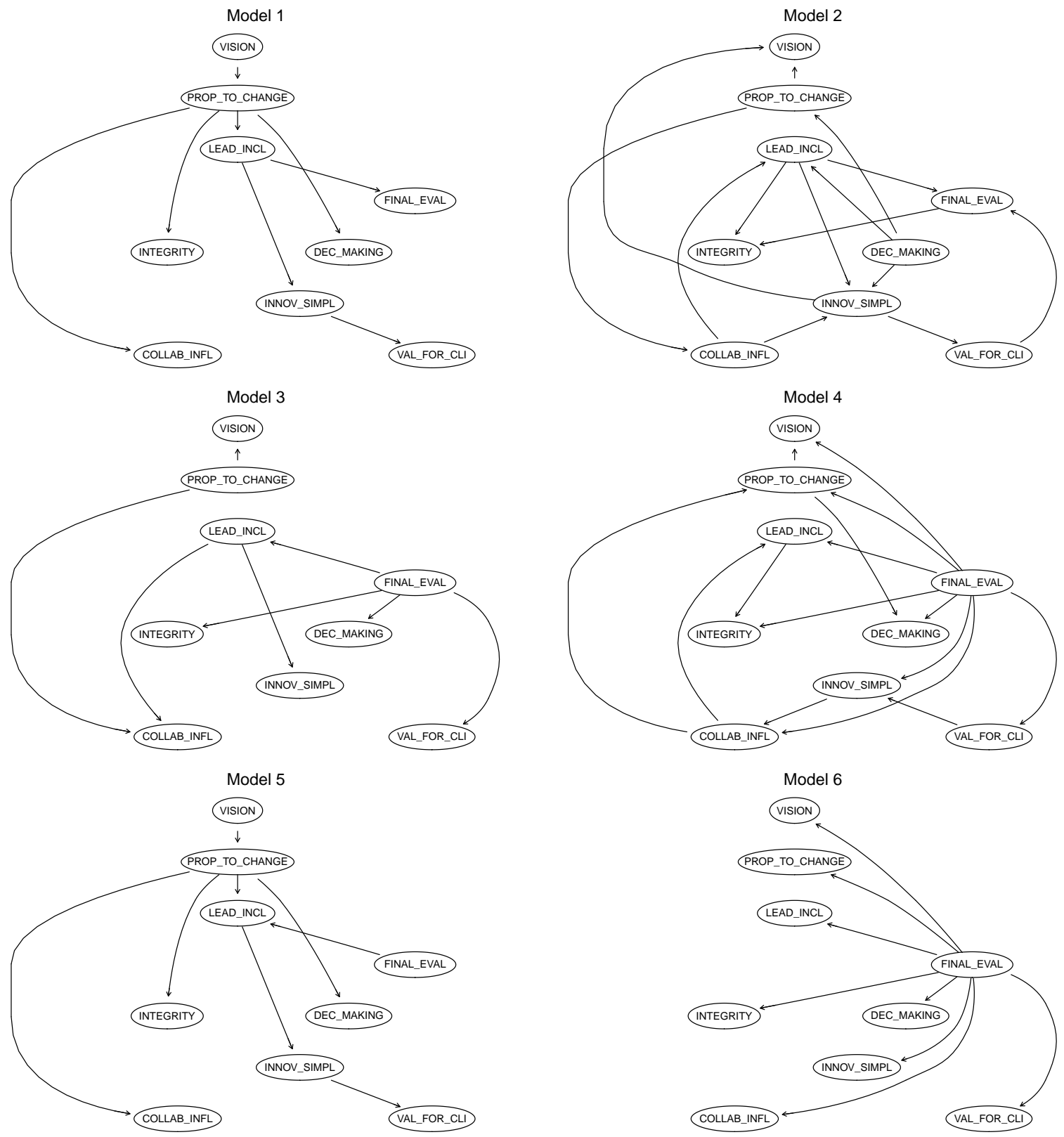

Figure 2: Different proposals for the connections between selected features. Here, we see hc (Model 1), bnsl (Model 2), chowliu (Model 3), tan (Model 4), hc mod (Model 5) and naive (Model 6). Notice how different algorithms sometimes switch direction in some connections (e.g. Vision and Propension to change): experts can decide to impose logical constraints, so to prescribe one of the two directions over the other, but in this specific use case both connections were just accepted as valid, because experts considered both conditioning as reasonable. 
Table 4: From Table 3, we can create an ordered rank among considered network structures: each model in the second column appears in the first column of next row, forming a chain of models in decreasing order of likelihood. Here, given the data, hc is more likely than bnsl, which is more likely than chowliu, etc.

\begin{tabular}{llr}
\hline model 1 & model 2 & $\log (\mathrm{BF})$ \\
\hline hc & bnsl & 5.437 \\
bnsl & chowliu & 86.692 \\
chowliu & naive & 4.401 \\
naive & tan & 0.899 \\
tan & hc_mod & 8.549 \\
\hline
\end{tabular}

Table 5: By comparing the sensitivity scores $\mathcal{S}(T, V)$ from (1), relative to different network structures, experts were able to disprove their belief that $V=$ Integrity was very important in determining the target $T=$ Final evaluation: in all structures, such variable had a limited impact on $T$.

\begin{tabular}{|c|c|c|c|}
\hline \multicolumn{2}{|l|}{ hc } & & \\
\hline \multirow{2}{*}{ variable } & \multirow{2}{*}{$\begin{array}{c}\text { sens. score } \\
\mathcal{S}(T, \cdot)\end{array}$} & \multicolumn{2}{|c|}{ chowliu } \\
\hline & & \multirow{2}{*}{ variable } & \multirow{2}{*}{$\begin{array}{c}\text { sens. score } \\
\mathcal{S}(T, \cdot)\end{array}$} \\
\hline LEAD_INCL & 0.581 & & \\
\hline PROP_TO_CHANGE & 0.296 & LEAD_INCL & 0.569 \\
\hline INNOV_SIMPL & 0.283 & DEC_MAKING & 0.491 \\
\hline COLLAB_INFL & 0.190 & VAL_FOR_CLI & 0.472 \\
\hline VISION & 0.183 & INNOV_SIMPL & 0.271 \\
\hline DEC_MAKING & 0.170 & INTEGRITY & 0.271 \\
\hline VAL_FOR_CLI & 0.142 & COLLAB_INFL & 0.112 \\
\hline INTEGRITY & 0.078 & & \\
\hline
\end{tabular}

was mostly influenced by Innovation and simplification, Integrity and Collaboration and ability to influence. However, most of the structures learned from the datasets suggest that, in fact, Integrity and Collaboration and ability to influence do not have a strong impact on the value of Final evaluation, while Inclusive leadership does. In Table 5 we show, side by side, the sensitivity score $\mathcal{S}(T, V)$, as defined in (1), for our target variable $T$ w.r.t. most relevant features $V$, based on the hc and chowliu network structures. As you can see, different DAG structures change the impact of different predictors, but some conclusions are shared across the DAGs.

Of course, this could be due to recent changes in the way such characteristics have been evaluated in the leadership model (do not forget that each feature in our dataset is not the direct measure of the skill of the auditor, but it represents the quintile which each auditor belonged to, based on its actual evaluation), or it could be due to some bias in the limited dataset. In any case, it is an information that experts were highly interested in, because one of their duties is to monitor in which ways evaluations change in time, so to spot any unwanted distorsions that could arise.

Notice that the usage of the sensitivity score (1) as a base for the considerations above is again motivated by the presence of categorical variables only in our model. When dealing with 


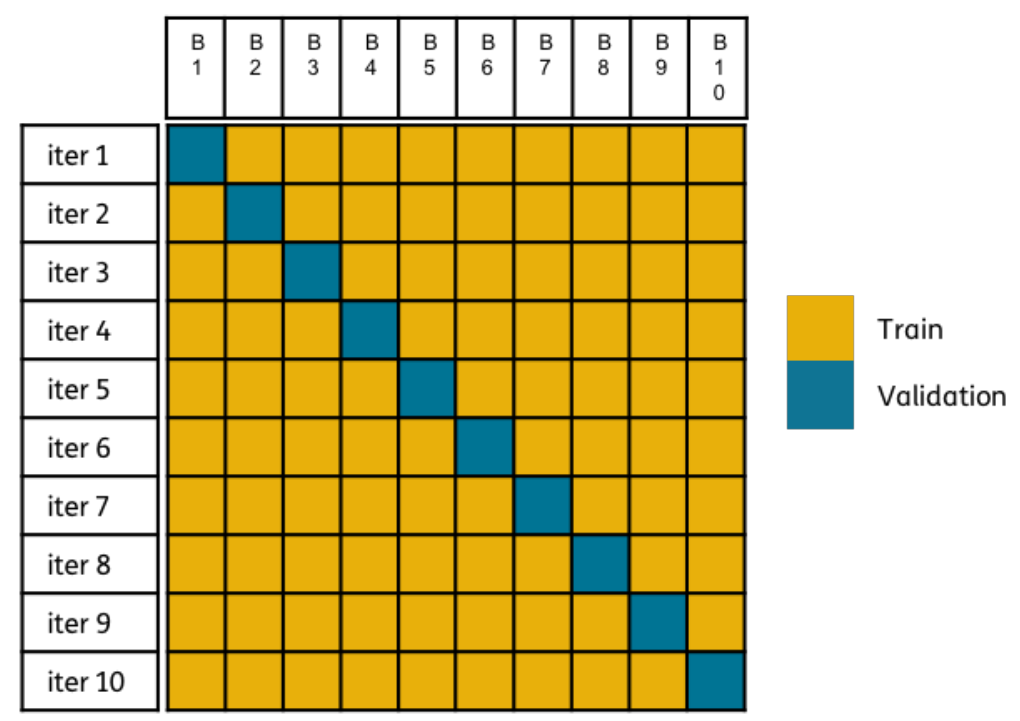

Figure 3: Cross validation scheme with 10 folds: each iteration of the cross validation procedure uses a different block as validation set and the remaining records as training set.

continuous variables and parameters, or with a mixture of continuous and discrete ones, information theoretical quantities might be harder to compute than in the example we considered here. Thus, when this is the case, one can use some alternative metric to compare the importance of variables in determining the predicted variables: for instance, by approximating the causal impact through partial dependency plots (assuming the model respects the underlying hypotheses), as in [22], or by investigating contributions through techniques like LIME [26] or SHAP values [27].

\subsection{Inference of parameters}

In the final step of our analysis, we want to choose the best model among the four we had previously selected. In order to compare them all and to decide which one better suits our needs, we set up a 10-fold cross validation procedure to measure average performances of each model on 10 different training/validation tests, as depicted in Figure 3.

In order to single out the best model and measure its predictive capabilities, we thus divide our dataset in two different chunks of row indices: a training set comprising $85 \%$ of the data and a test set comprising the remaining $15 \%$ of the records. The latter will be only used to evaluate the best model, once chosen, while the former will be extensively used in the cross validation procedure. Next, among the row indices of the training set, we select at random ten sets of 23 indices, i.e. $10 \%$ of the rows of the original dataset, for validation purposes: for each run, the records corresponding to such indices will form the validation sets, while the remaining data will be used as the actual training set for that run.

Hence, for each choice $i=1, \ldots, 10$, we perform training of the four models by using the $i$-th training set and we measure the performance on the $i$-th validation set. Here, both training and predictions are performed through MCMC simulation, following the ideas presented in Section 4.5 . Nodes are all assigned a Categorical distribution, with as many parameters as the dimension of the CPTs. These parameters are initially assumed to be distributed uniformly on the simplex with correct dimension (i.e. with $\operatorname{Dir}(\mathbf{1})$ for a vector $\mathbf{1}$ of suitable dimension) and get updated during training. For each record of the validation set, the model assigns as predicted 'label' for Final evaluation the state with larger probability. At the end of the cross validation procedure, we take 
Table 6: Performances of the four models included in the cross-validation procedure. In the evaluation table, we display both Accuracy (rate of correct classifications) and RMSE, but experts decided that the latter indicator was more useful because it also gave a measure of how far from correct predictions the model could go.

\begin{tabular}{lrr}
\hline Algorithm & Accuracy & RMSE \\
\hline chowliu & 0.413 & 0.769 \\
hc & 0.165 & 0.921 \\
bnsl & 0.204 & 1.019 \\
tan & 0.217 & 1.053 \\
\hline
\end{tabular}

Table 7: Summary of the performances for the selected model (chowliu) on the test set: even if the classifications are correct only in 1 out of 3 cases, the average error is smaller than 1 , and only in 3 cases the error in the prediction is larger than 1.

\begin{tabular}{rrrrr}
\hline Cases & Correct & Error $\geq 1$ & Accuracy & RMSE \\
\hline 35 & 12 & 3 & 0.3429 & 0.8783 \\
\hline
\end{tabular}

the average of the ten performance measures, in order to synthetize the results in a single score. In Table 6 we consider as measures:

- accuracy, meant as the proportion of predictions which are correct over the total number of predictions;

- root of mean square error ( $R M S E$ in the following), i.e. the mean of the Euclidean distance between the vector of predictions and the vector of actual values of the target variable.

Notice that, here, by accuracy we mean the elements on the diagonal of the confusion matrix for the classification problem (such a matrix has in position $(i, j)$ the number of records predicted of class $i$ by the model while being truly of class $j$ ).

In this use case, experts decided that RMSE was the most appropriate indicator of the two: indeed, we not only want a model that makes few mistakes, but among models with equal accuracy we prefer the one that, when wrong, is anyway as close as possible to the real value. As such mean square error is more desirable than accuracy alone, because it penalizes large mistakes. According to the comparison of the average RMSE, the best choice among the four models was Chow-Liu's algorithm, when equipped with conditional dependencies prescribed by experts, i.e. the structure we called chowliu (see "Model 3" in Figure 2).

Having finally chosen the model we want to use, we retrain it on the complete training set $(85 \%$ of the original data) and evaluate its overall performances on the test set (15\% of the original data). Results are shown in Table 7. Notice that, even if the model gives a wrong answer in about 2 out of 3 cases, it rarely deviates more than 1, meaning that the answer can be effectively used as a support to detect erroneous evaluation of auditors' job: even when the model makes mistakes, it is anyway very close to the correct answer.

One advantage of our Bayesian approach is that, in all stages, MCMC simulations produce complete distributions of the categorical target variable Final evaluation (cfr. Table 8), not only its most probable value. This gives additional "debugging" tools to experts, compared to 
Table 8: Posterior categorical distributions for Final evaluation, as computed by MCMC simulations, for a sample of records in the test set, together with the mean evaluation, the predicted value (i.e. the mode of the posterior) and the true value.

\begin{tabular}{lllllllllllll}
\hline 1 & 2 & 3 & 4 & 5 & 6 & 7 & 8 & 9 & 10 & mean & predicted & true \\
\hline 0.03 & 0.01 & 0.15 & 0.99 & 23.94 & 70.8 & 3.82 & 0.1 & 0.07 & 0.1 & 5.7813 & 6 & 5 \\
0.07 & 0.03 & 0.01 & 0.01 & 0.12 & 24.76 & 74.47 & 0.3 & 0.13 & 0.11 & 6.7532 & 7 & 6 \\
0.01 & 0.01 & 0.68 & 51.48 & 45.53 & 2.24 & 0.01 & 0.02 & 0.01 & 0.01 & 4.495 & 4 & 4 \\
0.01 & 0.01 & 0.79 & 51.29 & 45.69 & 2.13 & 0.02 & 0.04 & 0.01 & 0.01 & 4.4944 & 4 & 5 \\
$\cdots$ & $\cdots$ & $\cdots$ & $\cdots$ & $\ldots$ & $\cdots$ & $\cdots$ & $\cdots$ & $\cdots$ & $\cdots$ & $\cdots$ & $\cdots$ & $\cdots$ \\
\hline
\end{tabular}

Table 9: We show a few cases where consultants were in doubt due to apparently conflicting values of the predictors: experts would like to know how much they can trust the result given by the model. Thus, they look to the corresponding parameters of the distribution and observe that the model is way more convinced about its prediction in the first case, since state 5 has about $62 \%$ of probability to be the actual global evaluation, than in the second case, where $95 \%$ of probability is distributed across the values 4 to 6 , and 5 is only slightly more probable than 6 . Notice that, in this example, even the expected value of the distribution would not be of great help and only inspection of the posterior distributions allows to discriminate the two situations.

\begin{tabular}{rrrrrrrrrrrr}
\hline 1 & 2 & 3 & 4 & 5 & 6 & 7 & 8 & 9 & 10 & mean & predicted \\
\hline 0.03 & 0.03 & 1.02 & 8.95 & 61.86 & 27.19 & 0.69 & 0.10 & 0.07 & 0.06 & 5.1825 & 5 \\
0.03 & 0.04 & 1.86 & 25.37 & 35.99 & 34.77 & 1.67 & 0.18 & 0.03 & 0.06 & 5.0974 & 5 \\
\hline
\end{tabular}

typical pointwise estimates, since it offers a mean to verify how confident the model is about its predictions, or to compare the expected values with the true ones: if in a specific case the experts think the model might have made a mistake, they can inspect the corresponding distribution and react accordingly, see e.g. Table 9 .

We conclude this section by remarking that, in general, we expect the size of the split between training, validation and test sets to have a moderate impact on the performance of some of the models. In the current example, though, we saw no strong dependence on such a split, as long as enough records were kept for the training procedure: while minor variations in the specific values for RMSE were noticed, when varying the size of the test sets between $10 \%$ and $20 \%$ of the records, the ranking of the performances of different models stayed basically the same; when increasing the size of the test set to around $25 \%$ of the records, namely to 58 data entries, performances started to degrade for most models, due to underfitting because only $\tilde{1} 50$ records (65\% of the available data) were used for training and some of the dependencies were not properly learned.

\subsection{Details about MCMC simulation}

In the final stage of our analysis, we performed inference of the parameters of the chosen model via MCMC simulations. With the $\mathrm{R}$ packages described so far, there are two possible options to perform such a step, thanks to the discrete nature of the model considered in this specific case: on the one hand, we can use bnlearn functions bn.fit to first train the Bayesian network to the dataset, and then predict to perform predictions of Final evaluation on the test set; on the 
Table 10: Number of parameters associated to each node in the chosen Bayesian network (chowliu): the target feature has 10 possible states; other nodes have 5 states, but conditional dependences add dimensions to the CPTs.

\begin{tabular}{ll}
\hline node & \# of parameters \\
\hline FINAL_EVAL & 10 \\
PROP_TO_CHANGE & 5 \\
LEAD_INCL & $10 \times 5$ \\
INTEGRITY & $10 \times 5$ \\
VAL_FOR_CLI & $10 \times 5$ \\
DEC_MAKING & $10 \times 5$ \\
INNOV_SIMPL & $5 \times 5$ \\
VISION & $5 \times 5$ \\
COLLAB_INFL & $25 \times 5$ \\
\hline
\end{tabular}

other hand, we can write a JAGS model for the specific network structure chowliu, "Model 3" in Figure 2, and then to perform MCMC simulations through its R interface rjags [5] to both learn distribution parameters in each node and predict Final evaluation on the test set.

In both cases, we need to examine the behavior of the sampler (after the adaptation and burn-in phases of the algorithm), through trace plots and density plots of each parameter. Here, there would be plenty of parameters to inspect, as listed in Table 10, since each conditional dependence added to a node increases of a factor 5 the number of parameters to describe the Dirichlet distribution for that node (it is a factor 10 if the parent node is the target variable). We want to stress that, in general, the results of the inference might depend on the number of samples used in the simulation and in the number of Markov chains that are sampled. These are additional hyperparameters that should be accounted for in a more complex analysis, especially with continuous parameters appearing into the Bayesian structure. In our example, with categorical features only, we have seen no instabilities by using the default numbers of samples for adaptation and burn-in, and sampling more than three chains added no valuable insights to the inference.

In Figure 4, you can see the so called traceplot and densityplot for each parameter of the categorical distribution of Final evaluation: $\left(p_{1}, \ldots, p_{10}\right) \sim \operatorname{Dir}\left(\alpha_{1}, \ldots, \alpha_{10}\right)$, resulting from an MCMC simulation of our chowliu graph structure.

\section{Conclusions}

In this article, we presented a series of statistical tools that can be effectively used in corporate context, in order to guide business decisions and strategies. While each single element of the chain we used is well known in academic context, to our knowledge they are seldom used together, to the full extent of their capabilities, in industrial and strategic contexts. In presenting the specific experience developed by the authors in the past few years, we hope to have conveyed the wide range of possible applications of such methodology in different scenarios.

In Section 2, we described a specific corporate situation where the procedure leads to an effective support to domain experts in their job. In the case at hand, the model allowed to improve experts' beliefs, which were only partially confirmed about the importance of different features, as well as to receive an independent score against which to ponder their evaluation of human auditors. This is a general trend of application for data science tools, and for Bayesian models in particular: as human 

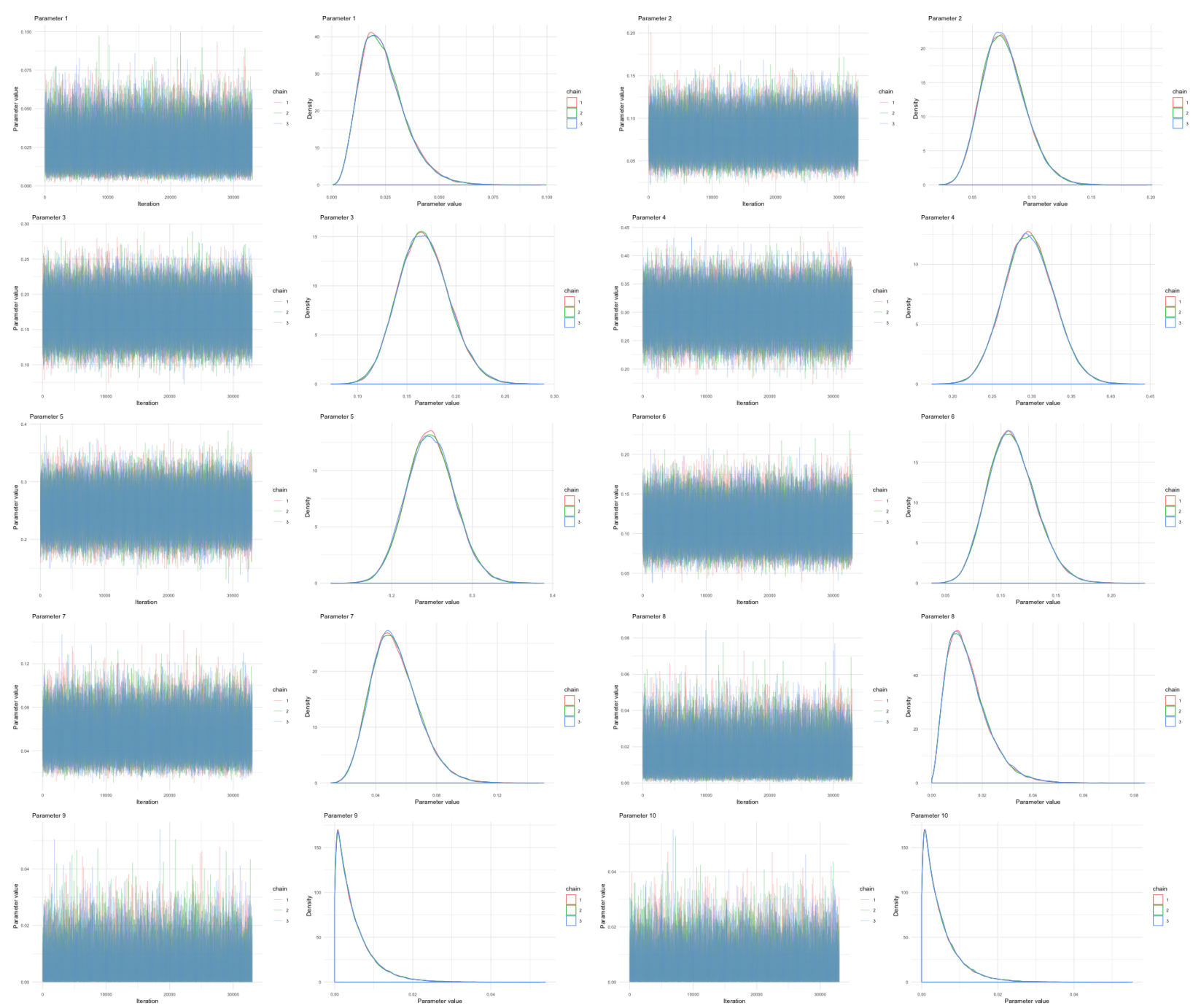

Figure 4: Trace and density plots for each of the ten parameters and each of the three Markov chains we used to approximate the posterior distribution for Final evaluation. You can see that the MCMC simulations have reached convergence, and that states 9 and 10 are judged highly improbable as one would expect given the limited available data. 
being, we are imperfect evaluator of uncertain scenarios and even worse synthethizers of different quantitative elements (especially due to heuristics and biases, see [25]). Hence, having consistent and coherent ways to aggregate and synthetize different sources of quantitative information, like the ones based on the Bayesian approach to uncertainty, could be of paramount importance towards a more effective course of actions in corporate decisions.

We would also like to stress the transparency value of the Bayesian techniques: in our context any manager could verify at any stage the effect of the measured predictors (skills and attitudes of auditors) on the target variable (global evaluation of auditors' job). In different contexts, the same transparency allows managers to monitor potential impacts of their strategies and to obtain a quantitative assessment that they can use to explain and communicate reasons behind their choices, e.g. to stakeholders. This is made possible because Bayesian networks allow to analyze the whole predictive posterior distribution, through suitable marginalization over the unobserved features, offering a more thorough measure of the impact of different features.

This is in stark contrast with most of the advanced machine learning algorithms used in industry: AI tools based on deep neural networks or (extreme) gradient boosting techniques, just to name two of the most popular algorithms, are typically "black boxes" that only provide a single prediction or probability, without any mean to unroll the sequence of evaluations which led the trained system to its result. Despite the recent efforts to develop local explainers, like the Local Interpretable Modelagnostic Explanations (or LIME) approach described in [26], or local and global SHAP values described in [27], the search for the reasons behind predictions given by black box algorithms is still way more difficult than in the case of Bayesian tools and it offers only partial results. The price to pay is in higher computational requirements than what would be needed for approximated measures like the aforementioned SHAP values [27], or partial dependence plots [22]. Nonetheless, we believe that the insights gained through the inspection of complete distributions is usually worth the effort and, thus, that the Bayesian approach can really represent an extremely powerful support towards more rational data-driven decisions in any corporate context.

Acknowledgement: The authors thank Prof. Julia Mortera for the fruitful discussions during the preparation of the paper. They also thank the anonymous referee for various suggestions which helped to improve the final revision of the paper.

\section{References}

[1] R Core Team. (2019). R: A language and environment for statistical computing. Vienna, Austria: R Foundation for Statistical Computing. Retrieved from https://www.R-project.org/

[2] Scutari, M. (2010). Learning Bayesian networks with the bnlearn R package. Journal of Statistical Software, 35(3), 1-22. doi:10.18637/jss.v035.i03

[3] Mihaljevi'c, B., Concha, B., \& Larranaga, P. (2018). Bnclassify: Learning Bayesian network classifiers. The $R$ Journal, 10(2), 455-468. Retrieved from 10.32614/RJ-2018-073

[4] Suzuki, J. (2017). An efficient Bayesian network structure learning strategy. New Generation Computing, 35, 105-124. doi:10.1007/s00354-016-0007-6

[5] Plummer, M. (2018). Rjags: Bayesian graphical models using mcmc. Retrieved from https: //CRAN.R-project.org/package=rjags

[6] Jaynes, E. T. (2003). Probability theory: The logic of science. Cambridge: Cambridge University Press. 
[7] Gelman, A., Carlin, J. B., Stern, H. S., \& Rubin, D. B. (2008). Bayesian data analysis (3rd ed.). Boca Raton, FL: Chapman; Hall/CRC.

[8] Kruschke, J. K. (2011). Doing Bayesian data analysis : A tutorial with $R$ and BUGS. Burlington, MA: Academic Press.

[9] Cowell, R. G., Dawid, A. P., Lauritzen, S. L., \& Spiegelhalter, D. J. (2007). Probabilistic networks and expert systems: Exact computational methods for Bayesian networks. New York, NY: Springer-Verlag.

[10] Pearl, J. (2009). Causality: Models, reasoning and inference (2nd ed.). New York, NY: Cambridge University Press.

[11] Hojsgaard, S., Edwards, D., \& Lauritzen, S. L. (2012). Graphical models with r. New York, NY: Springer-Verlag.

[12] Scutari, M., \& Denis, J.-B. (2014). Bayesian networks with examples in R. Boca Raton: Chapman; Hall.

[13] Margaritis, D. (2003). Learning Bayesian network model structure from data. Carnegie Mellon University.

[14] Verma, T., \& Pearl, J. (1991). Equivalence and synthesis of causal models. In Proceedings of the Sixth Annual Conference on Uncertainty in Artificial Intelligence (pp. 255-270). New York, NY, USA: Elsevier Science Inc.

[15] Cheng, J., Bell, D. A., \& Liu, W. (1997). An algorithm for Bayesian belief network construction from data. In Proceedings of AI ES STAT'97 (pp. 83-90).

[16] Metropolis, N., Rosenbluth, A. W., Rosenbluth, M. N., Teller, A. H., \& Teller, E. (1953). Equation of State Calculations by Fast Computing Machines. J. Chem. Phys., 21, 1087-1092. doi: $10.1063 / 1.1699114$

[17] Resnik, P., \& Hardisty, E. (2009). Gibbs sampling for the uninitiated. University of Maryland, Institute for Advanced Computer Studies (UMIACS).

[18] Vehtari, A., Gelman, A., \& Gabry, J. (2017). Practical Bayesian model evaluation using leave-one-out cross-validation and waic. Statistics and Computing, 27(5), 1413-1432. doi:10.1007/s11222-016-9696-4

[19] Fan, J., \& Lv, J. (2008). Sure independence screening for ultrahigh dimensional feature space. Journal of the Royal Statistical Society Series B, 70(5), 849-911. doi:10.1111/j.14679868.2008.00674.x

[20] Fan, J., Feng, Y. \& Song, R. (2011). Nonparametric Independence Screening in Sparse UltraHigh-Dimensional Additive Models. Journal of the American Statistical Association, 106, 544557. doi:10.1198/jasa.2011.tm09779

[21] Park, T. \& Casella, G. (2008). The Bayesian Lasso. Journal of the American Statistical Association, 103, 681-686. doi:10.2307/27640090

[22] Zhao, Q. \& Hastie, T. (2019). Causal Interpretations of Black-Box Models. Journal of Business E Economic Statistics, 2019, 1-19. doi:10.1080/07350015.2019.1624293 
[23] Friedman, N., Geiger, D., \& Goldszmidt, M. (1997). Bayesian network classifiers. Machine Learning, 29(2-3), 131-163. doi:10.1023/A:1007465528199

[24] Madsen, A. L., Lang, M., Kjærulff, U. B., \& Jensen, F. (2003). The hugin tool for learning Bayesian networks. In T. D. Nielsen \& N. L. Zhang (Eds.), Symbolic and quantitative approaches to reasoning with uncertainty (pp. 594-605). Springer Berlin Heidelberg. doi:10.1007/978-3-540-45062-7_49

[25] Kahnemann, D. (2011). Thinking, fast and slow. New York, NY: Farrar, Straus; Giroux.

[26] Ribeiro, M. T., Singh, S., \& Guestrin, C. (2016). "Why should i trust you?" Explaining the predictions of any classifier. In J. DeNero, M. Finlayson \& S. Reddy (Eds.), Proceedings of the 22nd ACM SIGKDD Conference on Knowledge Discovery and Data Mining, San Diego, CA. Association for Computational Linguistics, Stroudsburg, PA.

[27] Lundberg, S. \& Lee, S.-I. (2017). A Unified Approach to Interpreting Model Predictions. In I. Guyon, U.V. Luxburg et al. (Eds.), Advances in Neural Information Processing Systems 30 (NIPS 2017). Curran Associates, Inc., Red Hook, NY. (link to NIPS webpage)

\section{Appendix - Technical aspects}

In this section we focus on some technical ingredients which we have built our tools upon. They represent a selection of the rich catalogue of techniques for the so-called Bayesian inference and data analysis [6, 7, 8]: Bayesian networks, entropy and mutual information among variables, structure learning, Bayes-Turing factor and MCMC simulations.

Bayesian techniques are invaluable in corporate processes, because they allow to exploit all available information, usually quite sparse, and to synthetize it consistently with experts' opinions that can be modelled, not without considerable efforts, in forms of prior distribution of probability to be inserted into the model.

Since we are not adding new statistical developments to such tools and techniques, however, we recall here only some of them, for completeness sake. Readers, interested into general Bayesian networks and other topics briefly touched in this section, shall look into the aforementioned books and in [9, 10, 11].

\subsection{Bayesian networks}

A Bayesian network is a directed acyclic graph (DAG) whose nodes represent measurable features, and whose links between nodes represent mutual influences among variables. Since links are directed, such a structure creates a hierarchy among nodes and we will call parent nodes the starting points of such links and child nodes their ending points.

In order for any inference to take place, each variable has to be modelled in some way, i.e. a probability distribution of the variable has to be assumed, and each directed link is to be interpreted as a conditional dependence of the child variable on the parent one.

When dealing with discrete (or discretized) features only, as in the specific applications presented in Section 2, nodes of the graph are endowed with categorical distributions, and their parameters are assumed to be distributed according to suitable Dirichlet distributions. By combining structure learning algorithms (which will be presented afterwards) with domain experts' knowledge, it is then possible to figure out the most appropriate network structure and to impose prior distributions to the parameters. When models involve continuous features, like price or reliability estimates, the 
effort is higher because one needs some rigorous justification to impose the specific distributions. However, in the long run, we believe the resulting predictions definitely pay back the initial efforts, thanks to their explainability and to the production of a complete a posteriori distribution for each parameter. This, in turn, allows more thorough analysis of the phenomena, compared to what is possible with standard point estimates.

\subsection{Variable selection through mutual information}

The very first step to be performed, when building a model of the corporate process of interest, is the analysis of which features, among the available ones, are truly relevant in monitoring and controlling the process. In this section, we assume that available measures already monitor all aspects of interest and we focus our attention to the actual selection of relevant variables vs. redundant ones.

In our procedure, we choose to select relevant features by measuring the reciprocal influences between pairs of predictors, in a way that is independent of the model structure. As a metric to quantify such influences, we introduce the concept of mutual information, taken from information theory, and use such metric to decide which variables shall be kept and which discarded. In order to define such a quantity, we first borrow the concept of (Shannon) entropy : given a random variable $X$ taking discrete values in the set $\mathcal{X}$, we define its entropy as

$$
H(X)=-\sum_{x \in \mathcal{X}} p(x) \log p(x) .
$$

The idea beyond this quantity is to measure the amount of information about the distribution of $X$ that we can gather from the observation of evidences, given how probable each observation is: rarer events will provide more information; more common ones will provide much less information. A conditional version $H(X \mid Z)$ of the entropy can be defined analogously, for a pair of random variables $X$ and $Z$, by simply using the conditional distribution $p(x \mid z)$ in place of $p(x)$.

Once entropy has been defined, we can also give the following definition. If $X, Y$ are random variables supported on discrete sets $\mathcal{X}$ and $\mathcal{Y}$, we define mutual information MI among $X$ and $Y$ the quantity

$$
\operatorname{MI}(X, Y)=H(X)+H(Y)-H(X, Y),
$$

where $H(X, Y)$ is the entropy of the joint distribution of $(X, Y)$. Such a measure offers a few advantages compared to (Pearson's) correlation coefficient and its extensions: it gives a more appropriate measure of the relation between categorical variables $X$ and $Y$, whenever one of the features does not map well in terms of a numerical representations; it can be extended in a natural way to cope with the case of conditional probability distributions. This latter aspect plays a crucial role in selecting pairs of variables that can be combined to produce a Bayesian model of the corporate process.

Therefore, we will also define, for any triple of categorical variables $X, Y, Z$ taking values, respectively, in $\mathcal{X}, \mathcal{Y}, \mathcal{Z}$, the conditional mutual information $\mathrm{CMI}$ as follows

$$
\operatorname{CMI}(X, Y \mid Z)=H(X \mid Z)+H(Y \mid Z)-H(X, Y \mid Z)
$$

It is possible to give the same definition in presence of multiple conditioning on variables $Z_{1}, \ldots, Z_{k}$, by simply replacing the distributions conditional on $z \in \mathcal{Z}$ with those conditional on multiple variables.

However, there is still a detail to be accounted for: different pairs or triples of random variables cannot always be directly compared through their MI and CMI. When we want to compare different pairs of variables $X, Y$ and $Z, W$, we might end up with

$$
\operatorname{MI}(X, Y) \ll \operatorname{MI}(Z, W)
$$




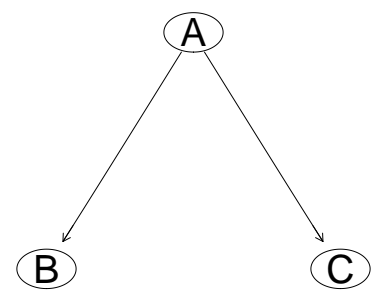

Figure 5: Features $B$ and $C$ could have effect on each other only when conditioned on their common parent $A$ : in view of the construction of a Bayesian network, it is important to explore conditional dependencies.

simply because variables $Z$ and $W$ have a different distribution compared to $X$ and $Y$, that gives them larger entropies, despite the influence of $Y$ on $X$ being possibly very important in the model of the process. This situation is quite common in corporate applications. For instance, you can think to a binary classification problems with rare class of interest (like in fraud or churn management processes): if other variables are distributed in a more uniform way, it might well be that scores involving the target variable can be order of magnitude smaller than scores not involving it. In order to cope with these cases, without the need of discriminating among variables with high and low entropy, we decided to replace $\operatorname{MI}(X, Y)$ from (3) and $\operatorname{CMI}(X, Y \mid Z)$ from (4) with their normalized versions 1

$$
\operatorname{MI}^{\prime}(X, Y)=2 \frac{\mathrm{MI}(X, Y)}{H(X)+H(Y)}, \quad \quad \mathrm{CMI}^{\prime}(X, Y \mid Z)=2 \frac{\mathrm{CMI}(X, Y \mid Z)}{H(X \mid Z)+H(Y \mid Z)} .
$$

With these measures at hand, the variable selection procedure can be carried out through a careful analysis of the quantities $\mathrm{MI}^{\prime}(X, Y)$ and $\mathrm{CMI}^{\prime}(X, Y \mid Z)$, as $X, Y, Z$ vary over all the available features. When variables have high $\mathrm{MI}^{\prime}$, they shall be considered as candidate connections in a Bayesian model. Also, pairs which have high $\mathrm{CMI}^{\prime}$ when conditional on a third variable, shall be evaluated in a Bayesian network as forming a graph structure as the one depicted in Figure 5. Finally, there might be value in the addition to the Bayesian model of triplets of variables $X, Y, Z$ which have $\mathrm{CMI}^{\prime}(X, Y \mid Z) \gg \mathrm{MI}^{\prime}(X, Y)$, especially when either of the variables $X, Y$ is of particular interest according to the experts of the corporate process.

Notice that similar analysis are exploited by structural learning algorithms for Bayesian networks (see e.g. [12, 13]) and they are used to analyse possible causal relationship in a polytree or a DAG (see [10]).

\subsection{Structure learning algorithms for Bayesian networks}

Once we have selected candidate features for inclusion in our Bayesian model, our next step focuses on inferring the actual structure of the model. This means to infer, from the available data, a DAG structure whose connections represent in the best possible way the relationship among predictors and targets. In order to perform such a step, we have to move beyond the local evaluation of dependence among pairs (or triplets) of variables and to compute the likelihood of our dataset of evidences, subject to the different DAGs which can connect the variables. Hence, in this section

\footnotetext{
${ }^{1}$ Sometimes $\mathrm{MI}^{\prime}$ is called symmetric uncertainty, or SU. By analogy, we could call $\mathrm{CMI}^{\prime}$ conditional symmetric uncertainty.
} 

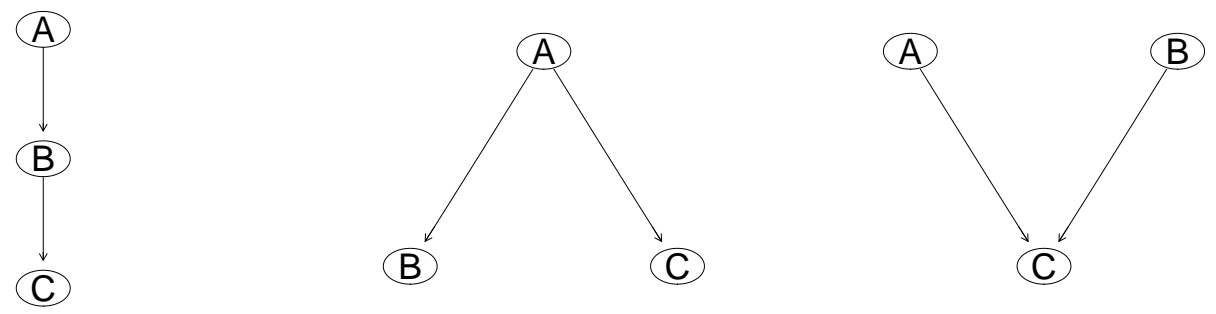

Figure 6: Different types of causal sub-structures that can be assigned by constraint-based structure learning algorithms.

we move our attention towards algorithms developed to perform structure inference, such as the ones described in [13] and implemented in some R packages like bnlearn [2, 12], bnclassify [3], and BNSL [4].

Despite a certain variety of theoretical motivations, most if not all such algorithms can be reduced to three broad categories: score-based algorithms, constraint-based algorithms, and hybrid algorithms.

The first category, score-based algorithms, approaches the problem by searching a DAG structure which maximizes a certain score measuring the posterior probability of each DAG structure $\mathcal{G}$ given the dataset $\mathcal{D}$. Since

$$
P(\mathcal{G} \mid \mathcal{D}) \propto P(\mathcal{D} \mid \mathcal{G}) P(\mathcal{G})
$$

most algorithms assume that $P(\mathcal{G})$ is uniform over a set of possible structures and concentrate on finding an approximate maximum of $P(\mathcal{D} \mid \mathcal{G})$. However, the whole space of admissible DAGs is too large to be fully explored and, typically, greedy search algorithms are used: one starts from a given initial network structure and it tries to modify it (by adding, deleting or reversing one arc at time), until there is no further change that improves the score. We will see the action of a score-based algorithm through the hc function of bnlearn.

Constraint-based structure learning algorithms, on the other hand, proceed along different lines. They were initiated by the work by Verma and Pearl [14] and attempt to:

- first identify which pairs of variables shall be connected by an arc, regardless of its direction,

- and then assign directions to arcs by identifying the correct $V$-structures among all nonadjacent pair of nodes with a common neighbor (cfr. Figure 6).

More precisely, in the former phase, algorithms of this kind test for the existence of connections by means of some independence test or information theory score (e.g. MI or $\mathrm{MI}^{\prime}$ as defined in (3) and (5), cfr. [15]), while in the latter phase they exploit conditional versions of the same test or score to search for the appropriate structures. We will see the action of a constraint-based algorithm through the chow.liu function of bnlearn.

Finally, hybrid structure learning algorithms have been proposed by combining aspects from constraint-based algorithms and aspect score-based ones. They typically proceed in two steps, called restrict and maximize: they start by finding a candidate set $\mathcal{P}_{i}$ of parents for each node $N_{i}$ (selected among nodes that are somehow 'related' to $N_{i}$ ), then they search the graph $\mathcal{G}$ which maximizes a given score, among structures that only contain edges connecting $N_{i}$ with nodes in $\mathcal{P}_{i}$. 


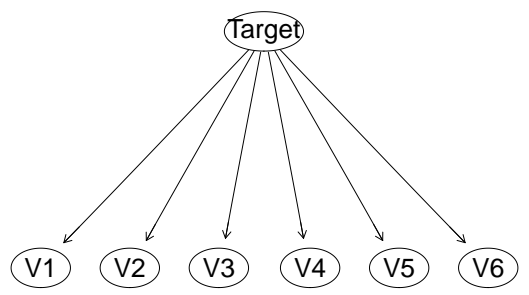

Figure 7: Naïve structure of Bayesian network: the target variable is the only parent node and all regressors are conditioned to it, while being (conditionally) independent from each other.

\subsection{Model selection through Bayes-Turing factor}

After applying the structure learning algorithms described in the previous section, we end up with many possible candidate models to describe the data-generating corporate process. Since our final goal consists of selecting a single model, which has captured most salient characteristics of the process and thus is capable to correctly predict future values of the target variables, we need a way to evaluate how well each model matches the available training data.

The approach we followed is the so-called Bayesian model selection, which builds a rank of the different models based on the Bayes-Turing factor (denoted by BF later on). Let our competing models be denoted by $\mathcal{H}_{1}$ and $\mathcal{H}_{2}$, and let $\mathcal{E}$ be the given set of evidences. Then, we define the Bayes-Turing factor as

$$
\operatorname{BF}\left(\mathcal{H}_{1}, \mathcal{H}_{2}\right)=\frac{P\left(\mathcal{E} \mid \mathcal{H}_{1}\right)}{P\left(\mathcal{E} \mid \mathcal{H}_{2}\right)}
$$

It is easy to see that, since $P\left(\mathcal{H}_{i} \mid \mathcal{E}\right) \propto P\left(\mathcal{E} \mid \mathcal{H}_{i}\right) P\left(\mathcal{H}_{i}\right), \mathrm{BF}\left(\mathcal{H}_{1}, \mathcal{H}_{2}\right)$ is exactly the factor that allows to update the ratio $P\left(\mathcal{H}_{1}\right) / P\left(\mathcal{H}_{2}\right)$ when new evidences are collected.

In our context, we exploit the following facts:

- a value $\operatorname{BF}\left(\mathcal{H}_{1}, \mathcal{H}_{2}\right)>1$ means that the evidences increase the chances of model $\mathcal{H}_{1}$ vs. model $\mathcal{H}_{2}$, compared to prior knowledge,

- a value $\operatorname{BF}\left(\mathcal{H}_{1}, \mathcal{H}_{2}\right)<1$ means that the evidences favors model $\mathcal{H}_{2}$ over model $\mathcal{H}_{1}$, compared to prior knowledge,

- a value $\operatorname{BF}\left(\mathcal{H}_{1}, \mathcal{H}_{2}\right)=1$ means that the evidences do not help discriminating among the models in any way.

By adopting BF in our workflow, we can effectively rank the different graph models suggested by structure learning algorithms and select the most promising one as the model to be used to support the decision making processes. Moreover, it becomes very easy to also add a naïve Bayes graph (cfr. Figure 7) in the ranking, i.e. a network where the target variables are parent nodes of all remaining features and no links are present among predictors. This simple structure acts as a reasonable benchmark to quantify how much the additional complexity, given by a non-naïve structure, actually pays off compared to the cheaper naïve model.

\subsection{Inference of parameters through MCMC simulations}

We got to the point where we have exploited our dataset to automatically select the variables with a major impact on the target variable, and to design the most likely DAG model for the datagenerating process. The result of this sequence of operations is a model of the corporate process, 

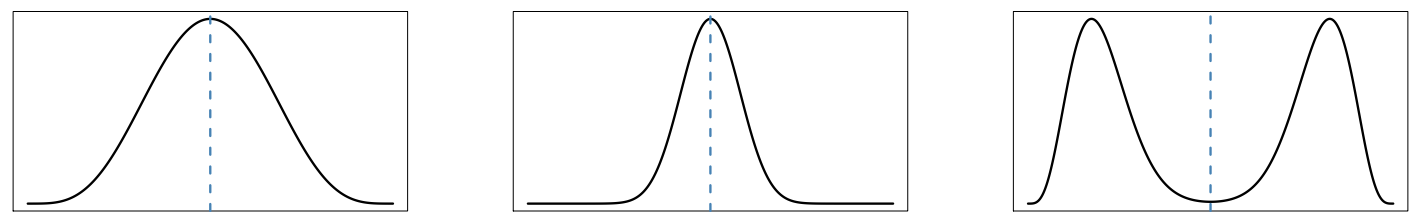

Figure 8: Some probability densities have differences that could not be summarized by a single pointwise estimate, e.g. the location of the mean of the posterior (dashed line).

depending on suitable parameters coming from the probability distributions associated to each node of the DAG. Our goal, at this stage, is to perform a Bayesian inference of all such parameters, so to obtain an estimate which incorporates both our prior beliefs and the evidences collected through data.

However, in general, the distributions in the model are not conjugate pairs of prior and likelihood, for which we could infer the parameters in closed analytical forms. This means that the posterior distributions has to be simulated via $M C M C$ methods. Such an approach, originated in 1950s with Metropolis-Hastings algorithm [16] with the goal of approximating distributions of particle collisions in Physics, allows to construct a sampler which produces collections of simulated data, whose distribution approximates accurately (on the long run) the underlying probability distribution.

In our corporate use case, this set of techniques allows to approximate very accurately the posterior distribution of the parameters, instead of obtaining only a pointwise estimator, like we could get with traditional maximal likelihood estimates $(M L E)$ or with a maximum a posteriori $(M A P)$ estimate. In turn, this enables experts from the corporate domain to judge by themselves how good a pointwise approximation would be, e.g. by discriminating among the distributions in Figure 8 .

We leave additional technical details to specific references in the Bibliography [7, 8, 17].

\subsection{Sequential usage of the different components}

Now that we have outlined separately each step of the methodological approach, it is time to summarize them all in a more comprehensive way.

Let us consider an hypothetical expert in a corporate environment, which is asked to decide the course of action to improve some prescribed KPI, based on a large dataset where several historical indicators have been recorded. According to the procedure streamlined in the previous sections, our expert will first perform a selection of the most significant predictors, based on $\mathrm{MI}^{\prime}$ and $\mathrm{CMI}^{\prime}$. Here, she will have a first chance to exploit her expertise into the process: specific features, deemed of interest, can be "manually" incorporated even if they obtained a low score, based on available data. This allows to include in later analysis both the experience of human decision makers and the signal present in the data, that could be driven by newer phenomena or by unexpected features of the process.

Then, the expert would feed the selected features into the component which performs structure learning, so to obtain different proposals of Bayesian models trained over the dataset. Here, she can prescribe the inclusion or exclusion of specific connections in the construction performed by algorithms, based on her judgement. In the end, by comparing of the Bayes-Turing factor (6) 
among pairs of different structures, she can select one or a few of them that seem valuable and that will be ultimately compared and ranked on the base of their actual predictive capabilities.

Finally, the chosen model can be trained and put in action, through MCMC simulations, so to predict future values of the target variable.

Notice that, in order to mitigate the risk of overfitting the training data with the structure inference and the parameter inference, one might want to perform the final selection through a $k$ fold cross validation procedure [18]. This is particularly useful in the case where multiple network structures offer similar performances at the end of the structure learning phase, i.e. they have a Bayes factor close to 1: the numerical simulations can then be used to evaluate more in details the generalization capabilities of each model, by selecting the structure which offers the best average performances among the different validation sets. The performance metric shall be chosen based on the specific problem at hand, either as a measure of the prediction errors or as a quantification of costs associated to predictions. More complex evaluation procedures could be set up as well (e.g. iterated cross validation, nested cross validation, etc.), when desired, but we do not account for them in this paper. 